\title{
Nonlinear $\delta$ F Simulation Studies of Intense Charged Particle Beams with Large Pressure Anisotropy*
}

\author{
Edward A. Startsev, Hong Qin, Ronald C. Davidson and W. Wei-li Lee \\ Princeton Plasma Physics Laboratory, Princeton University, Princeton, NJ 08543
}

\begin{abstract}
In this paper, a 3D nonlinear perturbative particle simulation code (BEST) is used to systematically study the stability properties of intense nonneutral charged particle beams with pressure anisotropy $\left(P_{\perp}>P_{\|}\right)$. The most unstable modes are identified and their eigenfrequencies and radial mode structure are determined for axisymmetric perturbations with $\partial / \partial \theta=0$.
\end{abstract}

\section{INTRODUCTION}

It's well known that in plasmas with strongly anisotropic distributions $\left(T_{|| b} / T_{\perp b} \ll 1\right)$ a collective instability may develop if there is sufficient coupling between the transverse and longitudinal degrees of freedom $[1,2,3]$. Such anisotropies develop naturally in accelerators, where the longitudinal temperature of the accelerated beam of charged particles with charge $q$ accelerated by a voltage $V$ is reduced according to $T_{\| b f}=T_{\| b i}^{2} / 2 q V$ ( for a nonrelativistic beam). At the same time, the transverse temperature may increase due to nonlinearities in the applied and selffield forces, nonstationary beam profiles, and beam mismatch. These processes provide the free energy to drive collective instabilities and may lead to a detoriation of beam quality.

\section{LINEAR THEORY}

Wang and Smith [2] investigated the kinetic stability properties of an intense particle beam assuming a $\mathrm{KV}$ distribution in the limit of large energy anisotropy $\left(T_{|| b} / T_{\perp b} \rightarrow 0\right)$ by expanding the solution of the linearized Vlasov equation in a series of Gluckstern eighenfunctions $\delta \varphi_{n}(r)=(1 / 2)\left[P_{n-1}\left(1-2 r^{2} / r_{b}^{2}\right)+P_{n}\left(1-2 r^{2} / r_{b}^{2}\right)\right]$, where $P_{n}(x)$ is the nth order Legendre Polynomial. The expansion yields a dispersion relation, expressible in terms of an infinite matrix determinant. For long-wavelength perturbations with $k_{z}^{2} r_{b}^{2} \ll 1$, one-half of the modes are identified as transverse $\left(T_{n}\right)$ Glucksteren modes with $\delta \varphi \propto \delta \varphi_{n}$. The other half consists of modes corresponding in the limit $\nu \rightarrow 0$ to an ordinary cold-beam longitudinal mode $\left(L_{1}\right)$ with $\delta \varphi \propto I_{0}\left(k_{z} r\right)$ inside the beam and $\omega^{2}=\left(\widehat{\omega}_{p b}^{2} / 2\right)\left(k_{z} r_{b}\right)^{2} \ln \left(r_{w} / r_{b}\right)$, plus a less-known class of "coupling" modes $\left(L_{n}\right)$ with $\delta \varphi \propto \delta \varphi_{n}$ and $\omega^{2}=\left[\widehat{\omega}_{p b}^{2} / 8 n(n+1)\right]\left(k_{z} r_{b}\right)^{2} \int_{0}^{2 \pi}(d x / 2 \pi) P_{n}(\cos x)$. The latter modes are the result of the interaction between transversely oscillating particles and the longitudinal perturbed

\footnotetext{
* Research supported by the U. S. Department of Energy
}

potential. Here, $\nu=\nu_{0}\left(1-s_{b}\right)^{1 / 2}$ is the depressed tune, where $s_{b}=\widehat{\omega}_{p b}^{2} / 2 \gamma_{b}^{2} \omega_{\beta b}^{2}$ is the normalized beam intensity, $\widehat{\omega}_{p b}^{2}=4 \pi \widehat{n}_{b} e_{b}^{2} / \gamma_{b} m_{b}$ is the plasma frequency-squared, $\nu_{0}=\omega_{\beta b}$ is the transverse betatron frequency associated with the applied focusing field, $r_{b}$ is the beam radius, and $r_{w}$ is the radius of the perfectly conducting wall.

As a general rule, for a KV distribution, instability arises in the regions of parameter space where two or more modes interact resonantly. The transverse modes $\left(T_{n}\right)$ are not significantly affected by longitudinal perturbations, and therefore the instability due to their interaction is a consequence of the fact that the KV distribution has a highly inverted population in phase space $[2,3,4]$. The most dangerous $T_{n}-L_{k}$ instabilities are due to $T_{2}-L_{1}$ interactions [2] in the region where $\nu / \nu_{0} \simeq 0.44$ with maximum growth rate $I m \omega / \nu_{0} \simeq 0.03$, and to $T_{2}-L_{2}$ interactions in the region $0.2 \leq \nu / \nu_{0} \leq 0.32$ with maximum growth rate $\operatorname{Im} \omega / \nu_{0} \simeq 0.15$. The latter mode has a much higher growth rate due to the similar transverse structure of the $L_{2}$ and $T_{2}$ modes. The growth rate is a maximum for $k_{z}^{2} r_{b}^{2} \geq 1$ in both cases.

To remedy the problem arising from an unphysical KV distribution, Davidson and Strasburg [1] employed a warmfluid model to investigate the stability properties of intense charged beams with pressure anisotropy[5]. The kinetic and fluid descriptions predict instability for different ranges of beam intensity. In the following section we compare these results with the simulation results using the nonlinear $\delta f$ simulation technique $[6,7]$.

\section{DESCRIPTION OF THE BEST NONLINEAR $\delta F$ SIMULATION CODE}

The theoretical models described in Sec. 2 use simplified assumptions for the background distribution. In practice, the transverse distribution function is close to thermal equilibrium with temperature $T_{\perp b}$, and the longitudinal distribution can be described by a drifting Maxwellian distribution with temperature $T_{\| b} \ll T_{\perp b}$. This distribution is stable with respect to transverse perturbations [4]. For an arbitrary equilibrium distribution one cannot solve the stability problem analytically and must employ numerical simulation techniques. To investigate stability properties numerically, we use the nonlinear $\delta f$ method [6] described below, as implemented in the Beam, Equilibrium, Stability and Transport (BEST) code [7].

In the smooth-focusing approximation, the transverse focusing force is modeled by $\mathbf{F}_{f o c}=-\gamma_{b} m_{b} \omega_{\beta b}^{2} \mathbf{x}_{\perp}$, where 
$\gamma_{b}=\left(1-\beta_{b}^{2}\right)^{1 / 2}$ is the relativistic mass factor, and $\beta_{b} c$ is the axial beam velocity. The solutions to the nonlinear Vlasov-Maxwell equations are expressed as $f_{b}=f_{b}^{0}+\delta f_{b}$, $\varphi=\varphi^{0}+\delta \varphi$ and $A_{z}=A_{z}^{0}+\delta A_{z}$, where $\left(f_{b}^{0}, \varphi^{0}, A_{z}^{0}\right)$ are known equilibrium solutions. The perturbed potentials satisfy the equations [7]

$$
\begin{gathered}
\nabla^{2} \delta \varphi=-4 \pi e_{b} \int d^{3} p \delta f_{b}, \\
\nabla^{2} \delta A_{z}=-\frac{4 \pi}{c} e_{b} \int d^{3} p v_{z} \delta f_{b},
\end{gathered}
$$

where $\delta f_{b}(\mathbf{x}, \mathbf{p}, t)$ is given by the weighted Klimontovich representation,

$$
\delta f_{b}=\frac{N_{b}}{N_{s b}} \sum_{i=1}^{N_{s b}} w_{b i} \delta\left(\mathbf{x}-\mathbf{x}_{b i}\right) \delta\left(\mathbf{p}-\mathbf{p}_{b i}\right) .
$$

Here, $N_{s b}$ is total number of beam simulation particles, $N_{b}$ is total number of actual beam particles, and the weight function is defined by $w_{b} \equiv \delta f_{b} / f_{b}$.

The nonlinear particle simulations are carried out by iteratively advancing the particle motion, including the weights they carry, according to

$$
\begin{gathered}
\frac{d \mathbf{x}_{b i}}{d t}=\left(\gamma_{b} m_{b}\right)^{-1} \mathbf{p}_{b i} \\
\frac{d \mathbf{p}_{b i}}{d t}=-\gamma_{b} m_{b} \omega_{\beta b}^{2} \mathbf{x}_{\perp_{b i}}-e_{b}\left(\nabla \varphi-\frac{v_{z b i}}{c} \nabla_{\perp} A_{z}\right) \\
\frac{d w_{b i}}{d t}=-\left(1-w_{b i}\right) \frac{1}{f_{b 0}} \frac{\partial f_{b 0}}{\partial \mathbf{p}} \cdot \delta\left(\frac{d \mathbf{p}_{b i}}{d t}\right) \\
\delta\left(\frac{d \mathbf{p}_{b i}}{d t}\right)=-e_{b}\left(\nabla \delta \varphi-\frac{v_{z b i}}{c} \nabla_{\perp} \delta A_{z}\right)
\end{gathered}
$$

and updating the fields by solving the perturbed Maxwell's equations with appropriate boundary conditions at the cylindrical, perfectly conducting wall at radius $r_{w}$. The $\delta f$ approach is fully equivalent to the original nonlinear Vlasov-Maxwell equations, but the noise associated with representation of the background distribution $f_{b 0}$ in conventional particle-in-cell (PIC) simulations is removed.

\section{SIMULATION RESULTS}

Here we present the simulation results for a continuous, anisotropic beam in a constant focusing field. The equilibrium distribution function is assumed to be

$$
\begin{gathered}
f_{0}\left(r, \mathbf{p}_{\perp}\right)=\frac{\widehat{n}_{b}}{\left(2 \pi \gamma_{b} m_{b}\right)^{3 / 2} \gamma_{b} T_{\perp b} T_{\| b}^{1 / 2}} \\
\times \exp \left\{-\frac{\left(p_{\|}-\gamma_{b} m_{b} \beta_{b} c\right)^{2}}{2 \gamma_{b}^{3} m_{b} T_{\| b}}\right\} \\
\times \exp \left\{-\frac{p_{\perp}^{2} / 2 \gamma_{b} m_{b}+\gamma_{b} m_{b} \omega_{\beta b}^{2} r^{2} / 2+e_{b}\left(\varphi_{0}-\beta_{b} A_{z 0}\right)}{T_{\perp b}}\right\},
\end{gathered}
$$

where $\widehat{n}_{b}$ is the beam density at $r=0$, and $T_{\perp b}$ and $T_{\| b}$ are the transverse and longitudinal temperatures of the beam particles. The equilibrium self-field potentials $\left(\varphi_{0}, A_{z 0}\right)$ are determined numerically from Maxwell's equations [7]. It is also assumed that the beam is located inside a grounded, cylindrical, perfectly conducting wall at radius $r_{w}=3 r_{b}$, where $r_{b}=\left[\left\langle r^{2}\right\rangle\right]^{1 / 2}$ is the rms beam radius. Random initial perturbations are introduced to the particle weights, and the beam is propagated from $t=0$ to $t=800 \omega_{\beta b}^{-1}$. The initial temperature ratio is taken to be $T_{\| b} / T_{\perp b}=0.04$. The simulations are performed for a wide range of normalized beam intensities ranging from $s_{b}=0.1$ to $s_{b}=0.95$, and detailed stability properties have been determined for the range of intensity parameters satisfying $s_{b} \geq 0.5$ assuming axisymmetric perturbations with $\partial / \partial \theta=0$. Shown in Fig. 1 is the time history of the density perturbation $\delta n_{b}=\int d^{3} p \delta f_{b}$ for normalized beam intensity $s_{b}=0.7$. After the initial linear growth, the instability saturates at the moderately large level $\left|\delta n_{b}^{\max } / \widehat{n}_{b}\right| \simeq 0.05$.

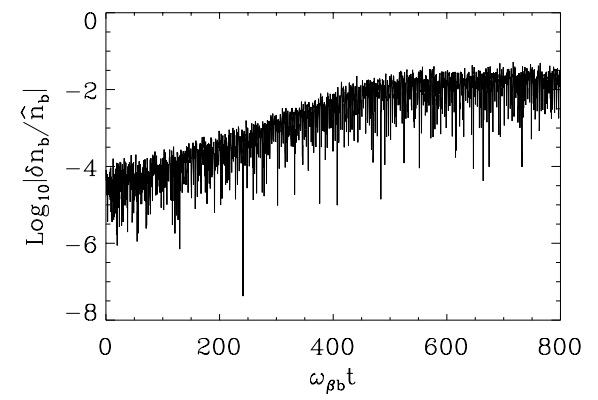

Figure 1: Time history of the normalized density perturbation $\delta n_{\max } / \widehat{n}_{b}$ for beam intensity $s_{b}=0.7$ at fixed $z$ and $r=0.2 r_{b}$.

The net change in the longitudinal momentum distribution $\delta F_{b}\left(p_{z}\right) / \widehat{F}_{0 b}$ at the conclusion of the simulation is shown in Fig.2. Here $\delta F_{b}\left(p_{z}\right)=\int d^{2} p_{\perp} d^{3} x \delta f_{b}$ and $\widehat{F}_{0 b}=\widehat{n}_{b} /\left(2 \pi \gamma_{b}^{3} m_{b} T_{|| b}\right)^{1 / 2}$. The formation of tails in axial momentum space in Fig. 2 and the consequent saturation of the instability are attributed to quasilinear stabilization. Figures 3 and 4 show plots of the real and imaginary parts

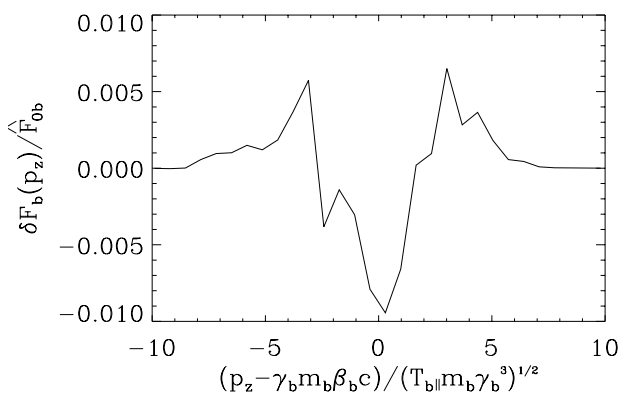

Figure 2: Perturbed longitudinal momentum distribution $\delta F_{b}\left(p_{z}\right) / \widehat{F}_{0 b}$ at time $t=800 \omega_{\beta b}^{-1}$, for normalized beam intensity $s_{b}=0.7$.

of the complex oscillation frequency $\omega$ versus normalized 
axial wavenumber $k_{z} r_{b}$. The instability has a finite bandwidth with maximum growth rate $\operatorname{Im} \omega / \omega_{\beta b} \simeq 0.02$ at $k_{z} r_{b}=2.5$. The unstable mode structure is shown in Fig.5

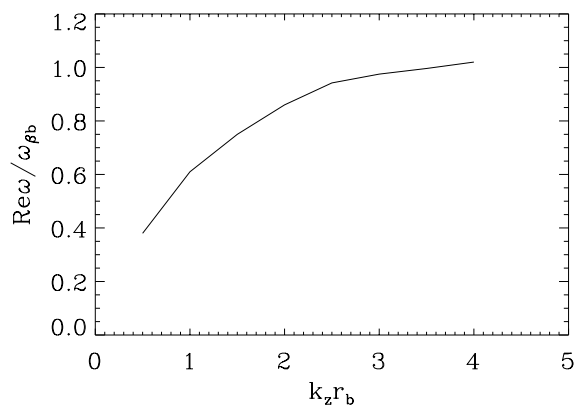

Figure 3: Normalized eigenfrequency $R e \omega / \omega_{\beta b}$ plotted versus $k_{z} r_{b}$ for $s_{b}=0.7$.

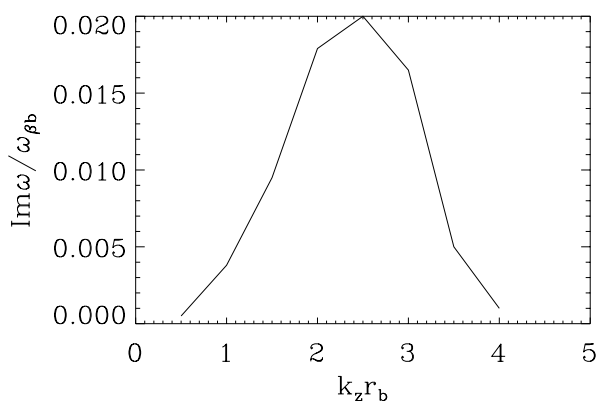

Figure 4: Normalized growth rate $\operatorname{Im} \omega / \omega_{\beta b}$ plotted versus $k_{z} r_{b}$ for $s_{b}=0.7$.

for $k_{z} r_{b}=2.5$. The dispersion relation and mode structure ( Fig.5) are similar to the well-known longitudinal $\left(L_{1}\right)$ mode for a cold beam [2]. For long wavelengths $k_{z}^{2} r_{b}^{2} \ll 1$, the dispersion relation is linear with Rew proportional to $k_{z} r_{b}$. For short wavelengths $k_{z}^{2} r_{b}^{2} \gg 1$, the transverse beam size is unimportant and $R e \omega \simeq 1.03 \omega_{\beta b}$. The de-

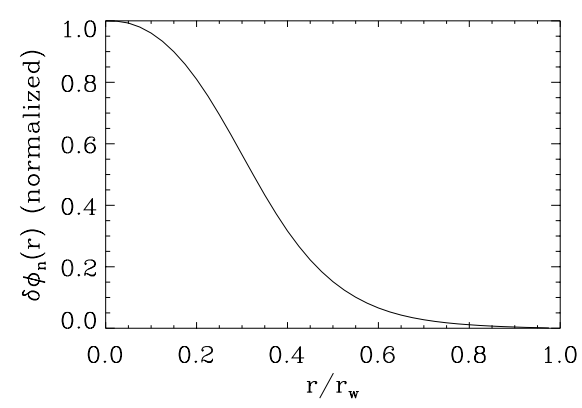

Figure 5: Radial mode structure of the unstable eigenfunction for $k_{z} r_{b}=2.5$ and $s_{b}=0.7$.

pendence of the maximum growth rate $(\operatorname{Im} \omega)_{\max } / \omega_{\beta b}$ on beam intensity $s_{b}$ is shown in Fig.6. The maximum growth rate $(\operatorname{Im} \omega)_{\max } / \omega_{\beta b} \simeq 0.038$ occurs for $s_{b} \simeq 0.8$, with no instability in the region $s_{b} \leq 0.5$.

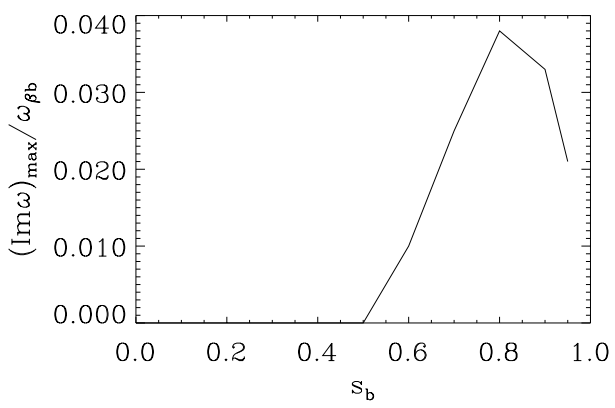

Figure 6: Plot of $(\operatorname{Im} \omega)_{\max } / \omega_{\beta b}$ versus normalized beam intensity $s_{b}$.

Finally, in the present simulations, the instability is found to be absent if the ratio of initial longitudinal and transverse temperatures is greater than the threshold value $T_{|| b} / T_{\perp b}=0.07$.

\section{CONCLUSIONS}

The BEST code [7], which implements the nonlinear $\delta f$ scheme, has been used to investigate the stability properties of intense charged particle beams with large temperature anisotropy $\left(T_{\| b} / T_{\perp b} \ll 1\right)$. The simulation results clearly show that moderately intense beams $s_{b} \geq 0.5$ are linearly unstable to short wavelength perturbations with $k_{z}^{2} r_{b}^{2} \geq 1$, provided the ratio of longitudinal and transverse temperatures is smaller than some threshold value. The mode structure, growth rate and the onset of the instability are qualitatively similar to what is predicted for a $\mathrm{KV}$ beam for the unstable $T_{2}-L_{1}$ mode interaction. In the nonlinear saturation stage, the total distribution function is still far from equipartitioned, and free energy is available to drive an instability of the hydrodynamic type [1].

\section{REFERENCES}

[1] R. C. Davidson and S. Strasburg, Phys. Plasmas 7, 2657 (2000).

[2] T. F. Wang and L. Smith, Part. Accel. 12, 247 (1982).

[3] S. M. Lund , et. al., Nucl. Instr. Meth. Phys. Res. A 415, 345 (1998).

[4] R. C. Davidson, Phys. Rev. Lett. 81, 991 (1999).

[5] S. M. Lund and R. C. Davidson, Phys. Plasmas 5, 3028 (1998).

[6] S. E. Parker and W. W. Lee, Phys. Fluids B 5, 77 (1993).

[7] H. Qin, R. C. Davidson and W. W. Lee, Physical Review Special Topics on Acceleration and Beams 3, 084401 (2000); 3, 109901 (2000). 